\title{
Cation size effects in oxygen ion dynamics of highly disordered pyrochlore-type ionic conductors
}

\author{
M. R. Díaz-Guillén, K. J. Moreno, J. A. Díaz-Guillén, and A. F. Fuentes \\ Cinvestav-Saltillo, Apartado Postal 663, 25000-Saltillo, Coahuila, Mexico \\ K. L. Ngai
Naval Research Laboratory, Washington, DC 20375-5320, USA \\ J. Garcia-Barriocanal, J. Santamaria, and C. Leon* \\ GFMC, Departamento de Fisica Aplicada III, Facultad de Fisica, Universidad Complutense de Madrid, 28040-Madrid, Spain
}

(Received 28 May 2008; revised manuscript received 29 August 2008; published 19 September 2008)

\begin{abstract}
In this work we evaluate the effect of cation size on the dc activation energy needed for oxygen ion migration, $E_{\mathrm{dc}}$, in highly disordered pyrochlore-type ionic conductors $A_{2} B_{2} \mathrm{O}_{7}$. Twenty six compositions with the general formula, $\mathrm{Ln}_{2} \mathrm{Zr}_{2-y} \mathrm{Ti}_{y} \mathrm{O}_{7}, \mathrm{Ln}_{1.7} \mathrm{Mg}_{0.3} \mathrm{Zr}_{2} \mathrm{O}_{7}\left(\mathrm{Ln}=\mathrm{Y}\right.$, Dy, and Gd), and $\mathrm{Gd}_{2-y} \mathrm{La}_{y} \mathrm{Zr}_{2} \mathrm{O}_{7}$, were prepared by mechanical milling, and their electrical properties were measured by using impedance spectroscopy as a function of frequency and temperature. By using the coupling model we also examine the effect of cation radii $R_{A}$ and $R_{B}$ on the microscopic potential-energy barrier, $E_{a}$, which oxygen ions encounter when hopping into neighboring vacant sites. We find that, for a fixed $B$-site-cation radius $R_{B}$, both activation energies decrease with increasing $A$-site-cation size, $R_{A}$, as a consequence of the increase in the unit-cell volume. In contrast, for a given $R_{A}$ size, the $E_{\mathrm{dc}}$ of the $\mathrm{Ln}_{2} \mathrm{Zr}_{2-y} \mathrm{Ti}_{y} \mathrm{O}_{7}$ series increases when the average $R_{B}$ size increases. This behavior is associated with enhanced interactions among mobile oxygen ions as the structural disorder increases with $R_{B}$.
\end{abstract}

DOI: 10.1103/PhysRevB.78.104304

PACS number(s): 66.10.Ed

\section{INTRODUCTION}

Oxides $A_{2} B_{2} \mathrm{O}(1)_{6} \mathrm{O}(2)$ with a pyrochlore structure have gained considerable attention in recent years because of their exceptionally high compositional diversity and structural flexibility. ${ }^{1-3}$ Combining different $A$ and $B$ cation pairs, pyrochlores electrical behavior span from that typical of insulators or semiconductors to materials showing high ionic, electronic, or mixed conductivity. ${ }^{4-6}$ The ideal pyrochlore cubic crystal structure (S.G. 227) might be derived from that of an anion deficient fluorite by doubling the unit cell, removing one out of every eight anions, and placing cations and anions in four crystallographically nonequivalent sites. Thus, $A\left(R_{A} \approx 1 \AA\right)$ and $B\left(R_{B} \approx 0.6 \AA\right)$ cations are, respectively, found at the $16 c$ (eight-coordinated) and $16 d$ (sixcoordinated) sites whereas anions are distributed between two tetrahedrally coordinated positions: $48 f[\mathrm{O}(1)]$ and $8 a$ $[\mathrm{O}(2)] .{ }^{7}$ There is in addition another tetrahedral site available for anions in the unit cell, $8 b$, which is systematically vacant in fully ordered pyrochlores that makes them poor oxygen ion conductors. However, defect pyrochlores such as $\mathrm{Gd}_{2} \mathrm{Zr}_{2} \mathrm{O}_{7}$, which are intrinsically disordered and exhibit the three aforementioned anion positions partially occupied, are good oxygen ion conductors at high temperatures. Different calculations have shown the most stable intrinsic defect in these compounds to be an oxygen Frenkel pair consisting of a vacant $48 f$ position and an interstitial ion located at the $8 b$ site. ${ }^{8-11}$ Thus, oxygen conductivity in pyrochlores depends essentially on the energy of formation of this defect. This energy is substantially reduced by the presence of disordering in the cation sublattice because cation disorder increases the similarity between nonequivalent oxygen sites and promotes Frenkel defect formation. Intriguingly, the most disordered pyrochlores are not necessarily the best oxygen ion conductors in the series because of higher activation energies $E_{\mathrm{dc}}$ needed for migration, and optimal conductivity is obtained in partially disordered materials. Atomic scale computing simulations have predicted the activation energy for oxygen migration in $A^{3+} / B^{4+}$ pyrochlores to be more dependent on the average size of cation $B$ than on that of $A .{ }^{8}$ For example, intermediate to large $B$ cations and intermediate size lanthanides ( $\mathrm{Sm}$ to $\mathrm{Er}$ ) in the $A$ position instead of the larger trivalent elements ( $\mathrm{La}$ to $\mathrm{Nd}$ ) should give the lowest $E_{\mathrm{dc}}$ for oxygen migration. However experimental data do not confirm clearly these predictions. Particularly, the effect of the cation size on the activation energy has not been completely understood since it will not only lead to changes in the unit-cell size but, as mentioned before, it will also produce changes in the degree of ordering/disordering of the pyrochlore crystal structure. In fact, we have recently reported that changes observed in the activation energy $E_{\mathrm{dc}}$ of the $\mathrm{Gd}_{2}\left(\mathrm{Ti}_{1-y} \mathrm{Zr}_{y}\right)_{2} \mathrm{O}_{7}$ solid solution when decreasing the $R_{\mathrm{Gd}} / R_{B}$ size ratio (increasing $\mathrm{Zr}$ content) might be explained by an increase in the cooperative effects among mobile ions as disorder increases. Interestingly, the activation energy $E_{a}$ for independent ion hopping remains almost constant in the series within experimental errors. ${ }^{12}$ The aim of the present contribution is to extend this analysis in exploring the role played by the average $A$ and/or $B$ cation sizes in the electrical properties of a large number of highly disordered pyrochlores. For this purpose, a wide set of $A_{2} B_{2} \mathrm{O}_{7}$ samples has been prepared (see Table I for a complete list) by combining different trivalent $\left(\mathrm{Y}^{3+}, \mathrm{Dy}^{3+}, \mathrm{Gd}^{3+}\right.$, and $\left.\mathrm{La}^{3+}\right)$ elements on the $A$ site, and $\mathrm{Zr}^{4+}$ and/or $\mathrm{Ti}^{4+}$ on the $B$ site. In addition, we have also included in the analysis some samples with $\mathrm{Mg}^{2+}$ partially substituted for $\mathrm{Ln}^{3+}$ to increase the number of oxygen vacancies, and find out its effect on the ionic conductivity and activation energy values. The electrical properties 
TABLE I. Composition and nomenclature of all samples analyzed in this work.

\begin{tabular}{lcc}
\hline \hline Nomenclature & Number of Samples & Chemical Formula \\
\hline$A Z$ & 3 & $A_{2} \mathrm{Zr}_{2} \mathrm{O}_{7}(A=\mathrm{Gd}, \mathrm{Y}, \mathrm{Dy})$ \\
$A \mathrm{MZ}$ & 3 & $A_{1.7} \mathrm{Mg}_{0.3} \mathrm{Zr}_{2} \mathrm{O}_{7}(A=\mathrm{Gd}, \mathrm{Y}, \mathrm{Dy})$ \\
$A \mathrm{MZT}$ & 3 & $A_{1.7} \mathrm{Mg}_{0.3} \mathrm{Zr}_{1.7} \mathrm{Ti}_{0.3} \mathrm{O}_{7}(A=\mathrm{Gd}, \mathrm{Y}, \mathrm{Dy})$ \\
$\mathrm{GLZ}$ & 5 & $\mathrm{Gd}_{2-x} \mathrm{La}_{x} \mathrm{Zr}_{2} \mathrm{O}_{7}(x=0.2 ; 0.3 ; 0.4 ; 0.8 ; 1)$ \\
$\mathrm{GZT}$ & 5 & $\mathrm{Gd}_{2} \mathrm{Zr}_{2-y} \mathrm{Ti}_{y} \mathrm{O}_{7}(y=0.2 ; 0.3 ; 0.7 ; 1.1 ; 1.3)$ \\
$\mathrm{DZT}$ & 3 & $\mathrm{Dy}_{2} \mathrm{Zr}_{2-y} \mathrm{Ti}_{y} \mathrm{O}_{7}(y=0.2 ; 0.6 ; 0.9)$ \\
$\mathrm{YZT}$ & 4 & $\mathrm{Y}_{2} \mathrm{Zr}_{2-y} \mathrm{Ti}_{y} \mathrm{O}_{7}(y=0.2 ; 0.7 ; 1.1 ; 1.3)$ \\
\hline \hline
\end{tabular}

were measured as a function of frequency and temperature by using impedance spectroscopy. Finally, contributions to the ion dynamics coming from interactions between mobile ions and from the change of the local barrier for oxygen ions hopping into neighboring vacant sites were analyzed as a function of the average $A$ and/or $B$ cation sizes.

\section{EXPERIMENT}

Samples were prepared by mechanically milling stoichiometric mixtures of high-purity elemental oxides, as described in previous works (see, for example, Refs. 13-15). Dry milling was carried out in a planetary ball mill by using zirconia vials and balls, and a moderate rotating disk speed (350 rpm). Phase composition of milled samples was analyzed by X-ray power diffraction (XRD) in a Philips X'Pert diffractometer using Ni-filtered $\mathrm{Cu} K_{\alpha}$ radiation $(\lambda=1.5418 \AA$ А $)$. The mechanically induced chemical reactions were considered completed when no traces of the starting reagents were observed by this technique. Admittance spectroscopy measurements were carried out on pellets $(10 \mathrm{~mm}$ diameter and $\sim 2 \mathrm{~mm}$ thickness) prepared by uniaxial pressing of the fine powders obtained by milling and sintering at a temperature of $1500{ }^{\circ} \mathrm{C}$. Both sides of the pellets were coated with colloidal $\mathrm{Pt}$ paint to act as blocking electrodes. Impedance data were recorded over the $100 \mathrm{~Hz}-1 \mathrm{MHz}$ frequency range as a function of temperature by using a Solartron 1260 frequency response analyzer.

\section{RESULTS AND DISCUSSION}

All samples analyzed in this work (see Table I) were found to display powder XRD patterns similar to that of either anion deficient fluorites or disordered pyrochlores. However, they are represented by the $A_{2} B_{2} \mathrm{O}_{7}$ chemical formula characteristic of the latter instead of the equivalent $(A, B)_{4} \mathrm{O}_{7}$ as nonstoichiometric fluorites. It has been argued that even in compounds such as $\mathrm{Y}_{2} \mathrm{Zr}_{2} \mathrm{O}_{7}$ (YZ), which are nominally fluorites, cation and anion distributions in their corresponding substructures are really never at random. ${ }^{16}$ Instead, they might be better represented by ordered pyrochlore-type microdomains in a disordered fluorite-type matrix. ${ }^{17}$ Therefore, for the purposes of this work they will not be considered "true" fluorite-type materials even if the superstructure reflections revealing the long-range ordering of cations and anion vacancies characteristic of pyrochlores (commonly of low intensity) (Ref. 18) are not detected by XRD (see Fig. 1).

Figure 2(a) shows a typical log-log representation of the frequency dependence of the real part of the electrical conductivity, $\sigma^{\prime}(\omega)$, for the $\mathrm{Y}_{2} \mathrm{Zr}_{2} \mathrm{O}_{7}$ sample over the $400{ }^{\circ} \mathrm{C}-800{ }^{\circ} \mathrm{C}$ temperature range. As observed in Fig. 2(a) at temperatures above $600{ }^{\circ} \mathrm{C}$, the conductivity value of this sample at each temperature is almost frequency independent with the high-frequency plateau identified as the dc conductivity, $\sigma_{\mathrm{dc}}$. Between $400{ }^{\circ} \mathrm{C}$ and $500{ }^{\circ} \mathrm{C}$, it is also evident that $\sigma^{\prime}(\omega)$ crosses over to a power-law dependence, $\sigma^{\prime}(\omega)$ $\propto \omega^{n_{J}}$, where $n_{J}$ is a fractional exponent $\left(0 \leq n_{J} \leq 1\right)$, at high frequencies. This behavior, called "universal dielectric response" (UDR) by Jonscher, ${ }^{19}$ has been linked to effects of

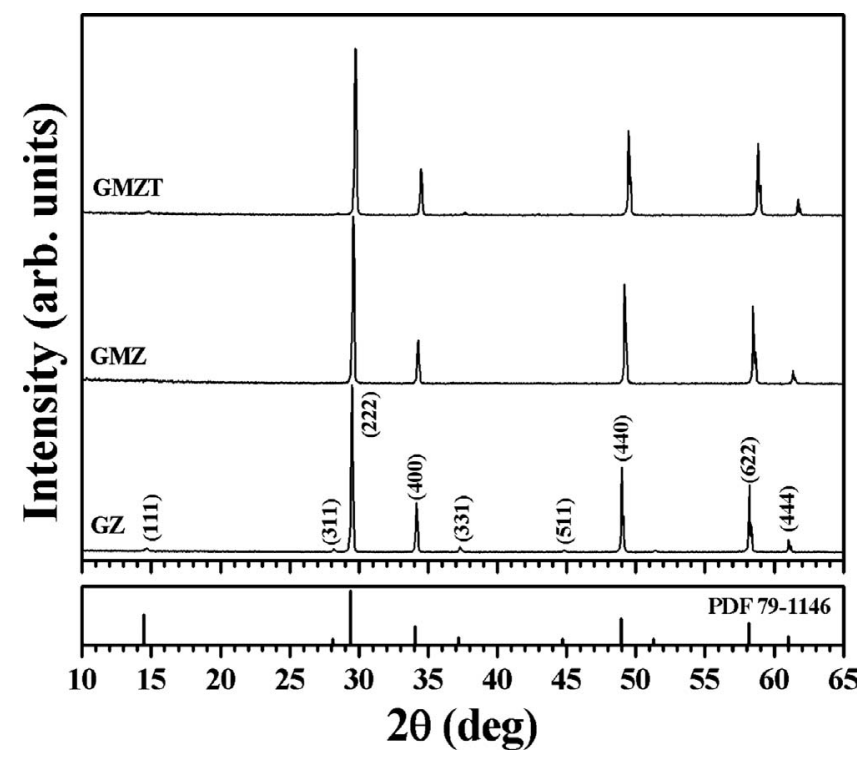

FIG. 1. XRD patterns obtained for selected samples, prepared as described in the Sec. II, after firing them at $1500{ }^{\circ} \mathrm{C}$ and slow cooling to room temperature. GZ, GMZ, and GMZT stand for $\mathrm{Gd}_{2} \mathrm{Zr}_{2} \mathrm{O}_{7}, \mathrm{Gd}_{1.7} \mathrm{Mg}_{0.3} \mathrm{Zr}_{2} \mathrm{O}_{7}$, and $\mathrm{Gd}_{1.7} \mathrm{Mg}_{0.3} \mathrm{Zr}_{1.7} \mathrm{Ti}_{0.3} \mathrm{O}_{7}$, respectively. Whereas the superstructure reflections characterizing the long-range ordering of cations and anion vacancies are barely evident in GZ and GMZT [e.g., (111) or (331) at $\sim 15^{\circ}$ and $37^{\circ}(2 \theta)$, respectively], they are apparently absent in GMZ, suggesting a different degree of structural disorder. As for comparison, the reported XRD pattern for pyrochlore-type $\mathrm{Gd}_{2} \mathrm{Zr}_{2} \mathrm{O}_{7}$ is also shown at the bottom. Numbers in parenthesis are the Miller indexes of each reflection. 

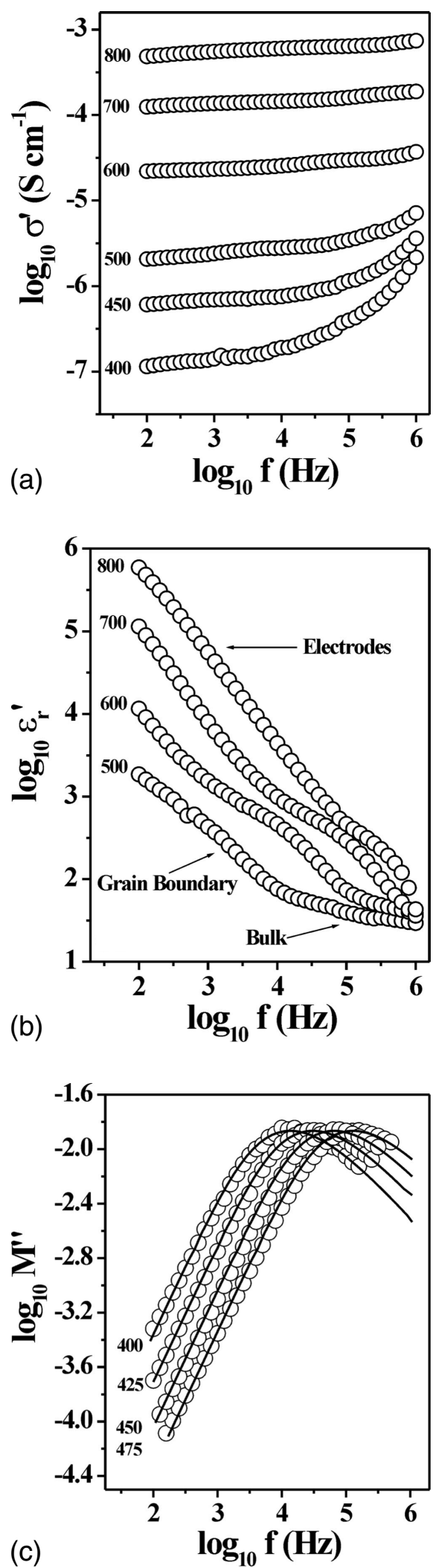

FIG. 2. Frequency dependence at selected temperatures of (a) the real part of conductivity, (b) the permittivity, and (c) the imaginary part of the electric modulus for $\mathrm{Y}_{2} \mathrm{Zr}_{2} \mathrm{O}_{7}$. Solid lines in (c) represent fits of the experimental data by $M^{\prime \prime}(f)$ calculated from the Fourier transform of a KWW correlation function. ion-ion correlation in the dynamics of hopping ions. ${ }^{20}$ In this interpretation, the value of $n_{J}$ monotonically decreases with decreasing interactions among mobile ions, and would be zero for completely independent and random ion hopping. Similar isothermal conductivity plots were obtained for the remaining samples analyzed in this work.

Figure 2(b) shows the frequency dependence of the real part of the permittivity for the same sample, also in a log-log representation. Blocking effects at grain boundaries and electrodes, which manifest as a large increase in the permittivity values toward low frequencies, are clearly evident and marked by labels in this figure. The presence of blocking effects, shifting toward higher frequencies with increasing temperature, indicate that the conductivity in the sample is basically ionic, as previously determined for these materials. ${ }^{6-12}$ Similar dielectric permittivity plots were obtained for the remaining samples. Thus, negligible electronic contribution to the overall conductivity is found in the samples used in this analysis, which is expected since we have avoided using compositions with very high $\mathrm{Ti}$ or $\mathrm{La}$ contents.

An alternative representation of the same electrical relaxation data (i.e., real and imaginary components of the sample impedance) is by using the complex electric modulus $M^{*}(\omega)$. The use of the electric modulus allows the obtaining of the relaxation function, $\Phi(\mathrm{t})$, in the time domain for the decay of the electric field inside the material under the constrain of a constant displacement vector. ${ }^{21,22}$ Under a constant displacement vector between the electrodes, ions will diffuse inside the material until a concentration gradient is created that cancels the electric field. Thus, the decay of the electric field [and its exact time dependence $\Phi(\mathrm{t})]$ is related to how ions can diffuse inside the material between the electrodes. It is found that the frequency dependence of the electric modulus is given directly by the Laplace transform of the time derivative of the relaxation function $\Phi(\mathrm{t})$, which is also the correlation function for ion hopping: ${ }^{21,22}$

$$
M^{*}(\omega)=\frac{1}{\varepsilon_{\infty}}\left[1-\int_{0}^{\infty}\left(-\frac{d \Phi}{d t}\right) e^{-j \omega t} d t\right],
$$

where $\varepsilon_{\infty}$ is the dielectric permittivity at high frequencies, and therefore the spectral shape and characteristic time of the electric modulus is determined by the dynamics of mobile ions, ${ }^{21}$ which we are interested in. The data of $\mathrm{Y}_{2} \mathrm{Zr}_{2} \mathrm{O}_{7}$, represented by the electric modulus, is shown in Fig. 2(c) by $M^{\prime \prime}(\omega)$, the imaginary part of $M^{*}(\omega)$. The $M^{\prime \prime}(\omega)$ data exhibit an asymmetric loss peak with maximum at a characteristic frequency $\omega_{p}$, which increases with increasing temperature. It is a common practice to use the Kohlrausch-WilliamsWatts (KWW) function, ${ }^{22}$

$$
\Phi(t)=\exp \left[-(t / \tau)^{1-n}\right],
$$

to represent the correlation function in Eq. (1), and the numerically calculated $M^{*}(\omega)$ to fit the experimental data. In Eq. (2), $\tau$ is the characteristic relaxation time of the ionhopping process and $(1-n)$ is a fractional exponent, e.g., 0 $<(1-n) \leq 1$. The fits are shown in Fig. 2(c) for the $\mathrm{Y}_{2} \mathrm{Zr}_{2} \mathrm{O}_{7}$ sample. The fractional exponent $n$ defines the power-law 


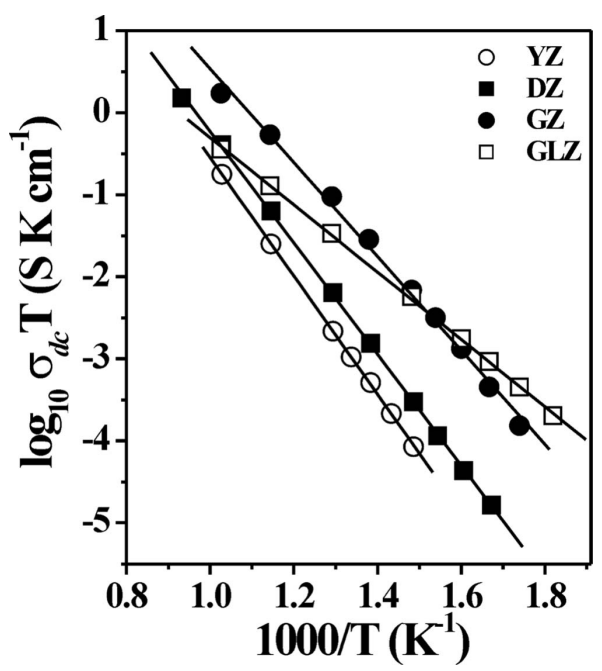

FIG. 3. Arrhenius plots of dc conductivity for several selected samples: YZ, DZ, GZ, and GLZ. Solid lines are the least-squares linear fits to the experimental data.

$\omega^{n-1}$ dependence of the $M^{\prime \prime}(\omega)$ at frequencies beyond the peak frequency $\omega_{p} \approx \tau^{-1}$, and also the aforementioned powerlaw dependence of the real part of the conductivity $\left[\sigma^{\prime}(\omega)\right.$ $\propto \omega^{n_{J}}$, with $\left.n_{J} \approx n\right]$ at higher frequencies. The best fits shown in Fig. 2(c) are in excellent agreement with our experimental data. From these fits, the values of the exponent $n$ were obtained and found to be temperature independent. The data of the other samples were analyzed in the same way, and the key parameters $n$ and $\tau$ were determined.

The temperature dependences of the dc conductivity for all samples were analyzed by using an Arrhenius-type law of the form $\sigma_{\mathrm{dc}}=\left(\sigma_{0} / T\right) \exp \left(-E_{\mathrm{dc}} / k T\right)$, where $E_{\mathrm{dc}}$ is the activation energy for the conduction process. Figure 3 shows the results in a plot of the product $\sigma_{\mathrm{dc}} T$ against reciprocal temperature for selected samples where the solid lines are least square fits to the experimental data, confirming in every case that the dc conductivity is thermally activated. The dc activation energy, $E_{\mathrm{dc}}$, calculated for each sample from the slope of these plots, was found to vary significantly from $0.78 \mathrm{eV}$ obtained for $\mathrm{Gd}_{2} \mathrm{Zr}_{0.7} \mathrm{Ti}_{1.3} \mathrm{O}_{7}$ to $1.43 \mathrm{eV}$ for $\mathrm{Y}_{1.7} \mathrm{Mg}_{0.3} \mathrm{Zr}_{2} \mathrm{O}_{7}$.

Two compatible models have been proposed to analyze electrical relaxation data in ionic conductors, ${ }^{23,24}$ which take into account the existence of the cooperative effects among mobile ions in the diffusion process. The coupling model (CM) (Ref. 24) starts with the consideration of independent hops of ions to vacant neighboring sites with exponential correlation function, $\Phi(t)=\exp \left(-t / \tau_{0}\right)$, and relaxation time $\tau_{0}$. Such independent hops cannot occur for all ions at the same time because of ion-ion interactions and correlations. The result of ion-ion interactions is the slowing down of the relaxation rate at times longer than $t_{c}$ of the order of $2 \mathrm{ps,}$ changing the correlation function from a pure exponential to a KWW function, wherein the value of the fractional exponent $n$ is a measure of the cooperative effects. A principal result from the CM is that the effective relaxation time $\tau$ is related to $\tau_{0}$ by

$$
\tau=\left[t_{c}^{-n} \tau_{0}\right]^{1 /(1-n)} .
$$

For ions vibrating in their cages and hopping to neighboring sites through barriers of energy $E_{a}$, the relaxation time for independent ion hopping is $\tau_{0}(T)=\tau_{\infty} \exp \left(E_{a} / k T\right)$. The reciprocal of $\tau_{\infty}$ is the attempt frequency of ions. It follows from Eq. (3) that the activation energy for the dc conductivity or $\tau$ will be larger than the actual energy barrier and the two are related by

$$
E_{\mathrm{dc}}=E_{a} /(1-n) .
$$

An increase in ion-ion interaction leads to a higher degree of cooperativity in the many-ion-hopping process, which corresponds to a higher value of $n$. Consequently, from Eq. (4), the activation energy for long-range ionic transport is higher due to the energy penalty that ion-ion interactions impose on the many-ionic diffusion process. The activation energy $E_{a}$, corresponding to the actual barrier that oxygen ions must overcome to hop (independently) between neighboring vacant sites in these highly disordered $A_{2} B_{2} \mathrm{O}_{7}$ pyrochlores, can be estimated according to Eq. (4) by using the experimental values obtained for $E_{\mathrm{dc}}$ and $n$.

Once the relevant parameters had been obtained as described above, the electrical properties of these samples were analyzed for their dependence on the $A$ and/or $B$-cation sizes by using the ionic radii given by Shannon. ${ }^{25}$ For this analysis, we have considered a common coordination number of eight for both positions $16 c$ and $16 d$ instead of eight and six, respectively, as in ideal pyrochlores, which is reasonable since all of them are present in a highly disordered anion sublattice. To facilitate the estimation of $R_{A}$ and $R_{B}$ values, we have also assumed no mixing between the $A$ and $B$ sites. When multiple occupancy of a single site, such as in $\mathrm{Gd}_{2} \mathrm{Zr}_{0.7} \mathrm{Ti}_{1.3} \mathrm{O}_{7}$, the average cation size, e.g., $R_{B}$, was calculated, taking into account also the fraction of component cations present.

As for the influence of the $B$-cation size, we have previously reported for $\mathrm{Gd}_{2} \mathrm{Zr}_{2-y} \mathrm{Ti}_{y} \mathrm{O}_{7}$ pyrochlores that there is a decrease in activation energy $E_{\mathrm{dc}}$ when increasing Ti content, which is essentially related to the decrease in the degree of correlations among mobile oxygen ions in the diffusion process as the structure becomes more ordered. ${ }^{12}$ This is in fact reflected in the concomitant decrease in the exponent $n$ characterizing conductivity relaxation dynamics according to the coupling model. ${ }^{12,21}$ In these sets of samples, structural disordering increases continuously as $\mathrm{Zr}$ content increases, ${ }^{14,26}$ and that is reflected in our results by the increase in ion-ion interactions and consequently in the value of $n$. Thus, we expect that for other lanthanides such as $\mathrm{Y}$ and Dy instead of $\mathrm{Gd}$ in the $A$ sites of the $\mathrm{Ln}_{2} \mathrm{Zr}_{2-y} \mathrm{Ti}_{y} \mathrm{O}_{7}$ systems, both the exponent $n$ and the activation energy $E_{\mathrm{dc}}$ also increase as $R_{B}$ increases ( $\mathrm{Zr}$ content increases) for fixed $R_{A}$. This is in fact what we observed as shown in Figs. 4(a) and 4(b). Note that the increase in the activation energy $E_{\mathrm{dc}}$ occurs despite increasing cell volume (e.g., $a=10.185 \AA$ vs $10.528 \AA$ for $\mathrm{Gd}_{2} \mathrm{Ti}_{2} \mathrm{O}_{7}$ and $\mathrm{Gd}_{2} \mathrm{Zr}_{2} \mathrm{O}_{7}$, respectively ${ }^{7}$ ), indicating that it the enhancement of ion-ion correlations that plays a dominant role in accordance with the coupling model predictions. Noticeably, as structural disordering in the Dy and Y containing series is higher than in the $\mathrm{Gd}$ one [both $\mathrm{Dy}_{2} \mathrm{Zr}_{2} \mathrm{O}_{7}$ (DZ) and $\mathrm{Y}_{2} \mathrm{Zr}_{2} \mathrm{O}_{7}$, are nominally fluorites at all temperatures 

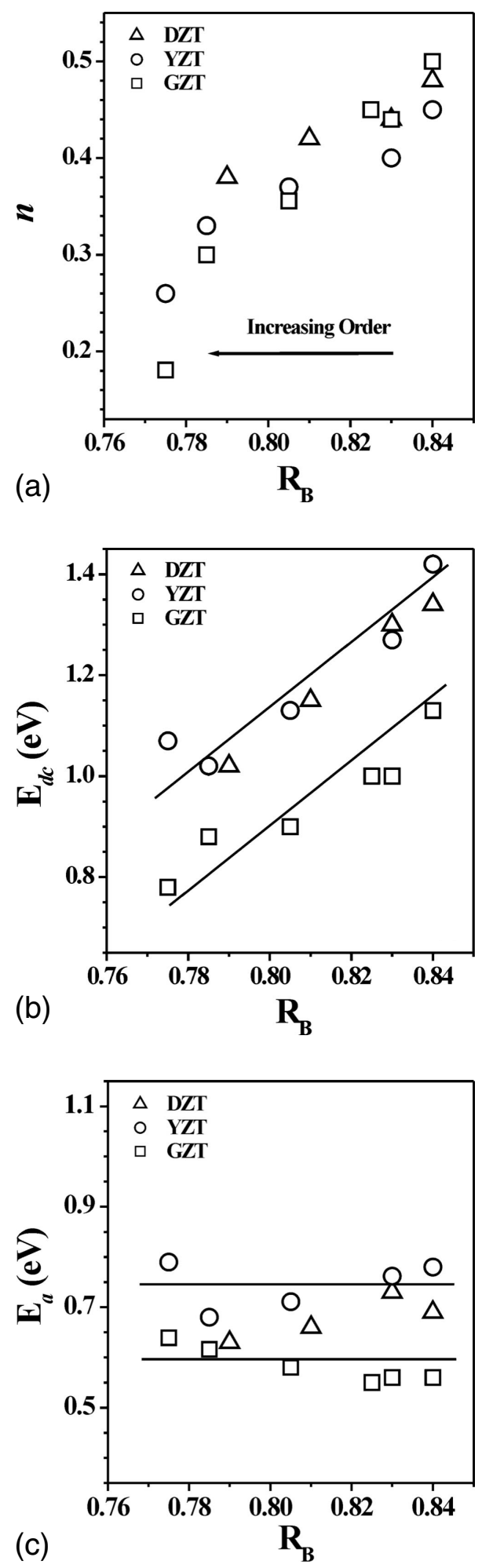

FIG. 4. Dependences of the values of (a) the exponent $n$, (b) the dc conductivity activation energy $E_{\mathrm{dc}}$, and (c) the $E_{a}$ on the average cation size $R_{B}$ for samples in the series of $\mathrm{Dy}_{2} \mathrm{Zr}_{2-y} \mathrm{Ti}_{y} \mathrm{O}_{7}$ (DZT), $\mathrm{Y}_{2} \mathrm{Zr}_{2-y} \mathrm{Ti}_{y} \mathrm{O}_{7}(\mathrm{YZT})$, and $\mathrm{Gd}_{2} \mathrm{Zr}_{2-y} \mathrm{Ti}_{y} \mathrm{O}_{7}$ (GZT), for which the value of $R_{A}$ cation radius remains fixed in each series. Note that the absolute scales in (b) and (c) are the same, and the solid lines are only shown to emphasize the trends. whereas $\mathrm{Gd}_{2} \mathrm{Zr}_{2} \mathrm{O}_{7}$ is pyrochlore below $1550{ }^{\circ} \mathrm{C}$ ], lower dc activation energies were obtained for the latter. As for the $E_{a}$, the energy barrier remains approximately constant for each series [see Fig. 4(c)], indicating that the difference between the observed $E_{\mathrm{dc}}$ and $E_{a}$ is indeed primarily due to the increase in slowing down of the oxygen ion-hopping dynamics by the many-ion cooperative effects. The degree of cooperativity in the dynamics of the mobile oxygen ions is enhanced by increasing $R_{B}$ (higher $\mathrm{Zr}$ content) since the mobile ion concentration increases by almost two orders of magnitude by increasing $\mathrm{Zr}$ content from $y=0$ to $1.5,{ }^{12}$ and thus naturally explains the increasing larger difference between $E_{\mathrm{dc}}$ and $E_{a}$.

It can be observed also in Fig. 4(c) that the average $E_{a}$ value decreases as $A$-site occupancy changes from Y to $\mathrm{Gd}$, accompanied by an increase in $R_{A}$. In order to confirm this behavior, we have plotted in Fig. 5(a) the evolution of the activation energy $E_{a}$ with the $A$-site average cation size for the samples in the $A_{2} \mathrm{Zr}_{2} \mathrm{O}_{7}(A Z), A_{1.7} \mathrm{Mg}_{0.3} \mathrm{Zr}_{2} \mathrm{O}_{7}$ (AMZ), and $\mathrm{Gd}_{2-x} \mathrm{La}_{x} \mathrm{Zr}_{2} \mathrm{O}_{7}$ (GLZ) zirconate series, all with constant $R_{B}\left[R_{\mathrm{Zr}}(\mathrm{VIII})=0.84 \AA\right]$. As this figure shows, $E_{a}$ systematically decreases when $R_{A}$ increases. This lower energy barrier for mobile oxygen ions might be explained by the increase in cell volume since it has been shown that, in fluorite and pyrochlore $\mathrm{Ln}_{2} \mathrm{Zr}_{2} \mathrm{O}_{7}$ lanthanide zirconates, cell volume is to a good approximation linearly dependent on the ionic radius of the $A$-type cation. $^{27}$ Therefore, on replacing $\mathrm{Gd}$ in $\mathrm{Gd}_{2} \mathrm{Zr}_{2} \mathrm{O}_{7}$ for $\mathrm{La}$, the unit-cell volume will increase ( $a$ $=10.528 \AA$ vs $10.805 \AA$ for $\mathrm{Gd}_{2} \mathrm{Zr}_{2} \mathrm{O}_{7}$ and $\mathrm{La}_{2} \mathrm{Zr}_{2} \mathrm{O}_{7}$, respectively ${ }^{7}$ ), and one would expect larger free space available for mobile charge carriers and, consequently, a lower barrier for oxygen ions to hop into neighboring vacant sites, consistent with the experimental data of GLZ series shown in Fig. 5(a). Since structural ordering/disordering is similar for all these pure zirconate samples, the value of the exponent $n$ obtained from the electric modulus vs frequency plots do not change significantly $(n=0.47 \pm 0.03)$, and therefore, the variation of the $E_{\mathrm{dc}}$ with the $A$-cation size is basically determined by the change in $E_{a}$. It is clear in Fig. 5(b) that this is the case and the trend is similar to that shown in Fig. 5(a). For comparison, we have also plotted in Fig. 5(a) as solid symbols the activation energy values for oxygen migration in $\mathrm{Gd}_{2} \mathrm{Zr}_{2} \mathrm{O}_{7}$ and $\mathrm{Y}_{2} \mathrm{Zr}_{2} \mathrm{O}_{7}$ obtained by Pirzada et al. ${ }^{8}$ by using atomic scale computer simulations; i.e., 0.58 and $0.65 \mathrm{eV}$, respectively. The existing structural disordering was not taken into account in these calculations by assuming in both cases a fully ordered pyrochlore structure. The aforementioned activation energy values from computer simulations would correspond in fact to the energy barrier, $E_{a}$, deduced from experimental data. As shown, the computer simulation value for $\mathrm{Gd}_{2} \mathrm{Zr}_{2} \mathrm{O}_{7}$ is quite similar to our result while that for $\mathrm{Y}_{2} \mathrm{Zr}_{2} \mathrm{O}_{7}$ is somewhat lower than the value we obtained. However, it should be recalled that yttrium zirconate $\mathrm{Y}_{2} \mathrm{Zr}_{2} \mathrm{O}_{7}$ does not exist as an ordered pyrochlore, as assumed in the computer simulations, ${ }^{8}$ but as an anion deficient fluorite, and as pointed out by Van Dijk et al., ${ }^{28}$ activation energies for oxygen migration are significantly higher in the latter, which would explain the difference of activation energies from simulation and experiment for $\mathrm{Y}_{2} \mathrm{Zr}_{2} \mathrm{O}_{7}$.

Thus, we find that the experimental activation energy $E_{\mathrm{dc}}$ for the dc conductivity due to the mobile oxygen ions in 



FIG. 5. The activation energies (a) $E_{a}$ and (b) $E_{\mathrm{dc}}$ for selected samples, with fixed $R_{B}$ cation size, as a function of the average $R_{A}$ cation radius. $A Z, A M Z$, and GLZ stand for $A_{2} \mathrm{Zr}_{2} \mathrm{O}_{7}$, $A_{1.7} \mathrm{Mg}_{0.3} \mathrm{Zr}_{2} \mathrm{O}_{7}(A=\mathrm{Y}$, Dy and $\mathrm{Gd})$, and $\mathrm{Gd}_{2-x} \mathrm{La}_{x} \mathrm{Zr}_{2} \mathrm{O}_{7}$ samples, respectively. Dashed lines are not linear fits and are only shown to emphasize the drop in both activation energies as the size of the $A$-site cation increases. The solid diamond and solid circle symbols in (a) represent activation energy values for oxygen migration in $\mathrm{Gd}_{2} \mathrm{Zr}_{2} \mathrm{O}_{7}$ and $Y_{2} \mathrm{Zr}_{2} \mathrm{O}_{7}$, respectively, calculated by atomic scale computer simulations (Ref. 8).

these pyrochlores increases with $R_{B}$ and decreases with $R_{A}$. Figure 6 shows that there is indeed a correlation between $E_{\mathrm{dc}}$ values obtained for every sample analyzed in this work with the ratio $R_{A} / R_{B}$ of the average cation sizes. It becomes very clear that, despite the differences in chemical composition and even the increasing concentration of vacancies $(\mathrm{Mg}-$ doped samples), the $E_{\mathrm{dc}}$ follows a general trend and decreases sharply as the size ratio increases, and the size mismatch between cations at the $A$ and $B$ sites increases. For example, the $E_{\mathrm{dc}}$ almost decreases by a factor of two by dropping from $1.43 \mathrm{eV}$ for $\mathrm{Y}_{1.7} \mathrm{Mg}_{0.3} \mathrm{Zr}_{2} \mathrm{O}_{7}\left(R_{A} / R_{B}=1.19\right)$ to $0.78 \mathrm{eV}$ for $\mathrm{Gd}_{2} \mathrm{Zr}_{0.7} \mathrm{Ti}_{1.3} \mathrm{O}_{7}\left(R_{A} / R_{B}=1.36\right)$. This trend persists across the theoretical fluorite/pyrochlore phase boundary, which for zirconates and titanates has been estimated at a $R_{A} / R_{B}$ value corresponding to that of $\mathrm{Gd}_{2} \mathrm{Zr}_{2} \mathrm{O}_{7}$ between

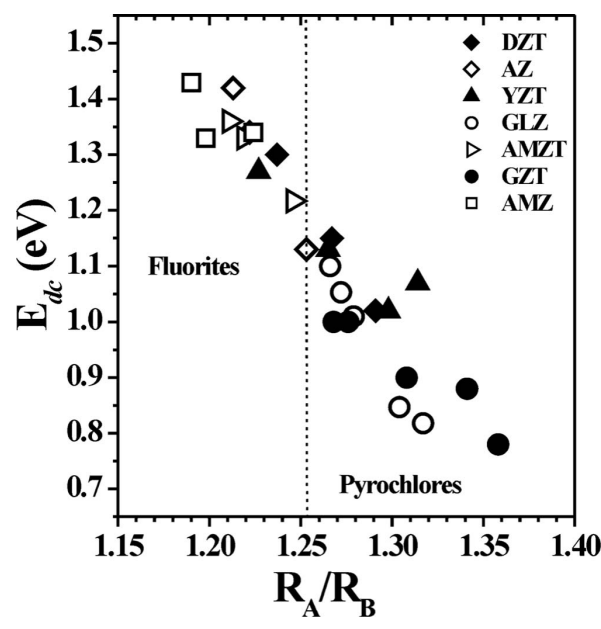

FIG. 6. dc conductivity activation energies for all samples studied, plotted as a function of the average cation size ratio $R_{A} / R_{B}$. Vertical dashed line represents the estimated fluorite/pyrochlore phase boundary for titanates and zirconates.

1.25 and 1.26 (Ref. 7) (the structure is fluorite when the size ratio falls below these values and pyrochlore otherwise). In agreement with the results previously discussed in Figs. 4 and 5, the correlation between $E_{\mathrm{dc}}$ and the ratio $R_{A} / R_{B}$ shown in Fig. 6 can be understood as a consequence of two simultaneous mechanisms working together in the same direction. On the one hand, the available cell volume for oxygen hopping increases by increasing the $R_{A} / R_{B}$ ratio and hence decreasing the dc activation energy for oxygen diffusion. On the other hand, structural disordering increases as the $R_{A} / R_{B}$ ratio decreases toward one (both cations having a more similar size), which leads to enhanced ion-ion interactions in the diffusion process, and hence to higher activation energy for the dc conductivity.

\section{SUMMARY AND CONCLUSIONS}

We have analyzed twenty six ionically conducting zirconate-titanate disordered pyrochlores $\left(A_{2} B_{2} \mathrm{O}_{7}\right)$, which are of different composition, to investigate the effect of cation ionic radii $R_{A}$ and $R_{B}$ on the dynamics of mobile oxygen ions. Impedance spectroscopy measurements allowed us to obtain for each sample the activation energy for the dc conductivity, $E_{\mathrm{dc}}$, and the value of the exponent $n$, characterizing conductivity and electric modulus spectra. From these values we have obtained the actual energy barrier for oxygen ion hopping according to the coupling model. Our results show that there is a systematic increase in both $n$ and $E_{\mathrm{dc}}$ when increasing $R_{B}$ for a given fixed $R_{A}$ due to enhanced ion-ion interactions by increasing structural disorder and the number of mobile ions. On the other hand, for the pure zirconate samples that have a fixed $R_{B}$ value and a high degree of structural disorder, there is a significant decrease in the activation energy $E_{\mathrm{dc}}$ for oxygen diffusion when the value of $R_{A}$ is increased. This can be understood in terms of the increasing cell volume when increasing $R_{A}$ because this would facilitate oxygen ion mobility by lowering the energy barrier for hopping between neighboring sites. We have also found 
that all our results can be rationalized by the coupling model predictions. There is also support of this rationalization by the results of previous atomic scale computer simulations. Most interestingly, the correlation found between the activation energy $E_{\mathrm{dc}}$, limiting the ionic conduction process, and the cation size ratio $R_{A} / R_{B}$ might help in the search of materials with higher ionic conductivity values at room or moderately higher temperature.

\section{ACKNOWLEDGMENTS}

This work has been carried out with the financial support of Mexican Conacyt (Grant No. SEP-2003-C02-44075) and Spanish MCYT (Contracts No. MAT2005-06024-C02 and No. MAT2008-06517-C02). M.R.D.G. thanks Conacyt for financial support. K.L. Ngai was supported in part by ONR under Program Element and Project 61153N.
*Corresponding author. carletas@ fis.ucm.es

${ }^{1}$ A. P. Ramirez, A. Hayashi, R. J. Cava, R. Siddharthan, and B. S. Shastry, Nature (London) 399, 333 (1999).

${ }^{2}$ J. Snyder, J. S. Slusky, R. J. Cava, and P. Schiffer, Nature (London) 413, 48 (2001).

${ }^{3}$ W. J. Weber and R. C. Ewing, Science 289, 2051 (2000).

${ }^{4}$ O. Porat, C. Heremans, and H. L. Tuller, Solid State Ionics 94, 75 (1997).

${ }^{5}$ E. V. Tsipis, V. V. Kharton, and J. R. Frade, J. Eur. Ceram. Soc. 25, 2623 (2005).

${ }^{6}$ S. A. Kramer and H. L. Tuller, Solid State Ionics 82, 15 (1995).

${ }^{7}$ M. A. Subramanian, G. Aravamudan, and G. V. Subba Rao, Prog. Solid State Chem. 15, 55 (1983).

${ }^{8}$ M. Pirzada, R. W. Grimes, L. Minervini, J. F. Maguire, and K. E. Sickafus, Solid State Ionics 140, 201 (2001).

${ }^{9}$ P. J. Wilde and C. R. A. Catlow, Solid State Ionics 112, 185 (1998).

${ }^{10}$ R. E. Williford, W. J. Weber, R. Devanathan, and J. D. Gale, J. Electroceram. 3, 409 (1999).

${ }^{11}$ P. J. Wilde and C. R. A. Catlow, Solid State Ionics 112, 173 (1998).

${ }^{12}$ K. J. Moreno, G. Mendoza-Suárez, A. F. Fuentes, J. GarcíaBarriocanal, C. León, and J. Santamaría, Phys. Rev. B 71, 132301 (2005).

${ }^{13}$ K. J. Moreno, R. Silva-Rodrigo, and A. F. Fuentes, J. Alloys Compd. 390, 230 (2005).

${ }^{14}$ K. J. Moreno, M. A. Guevara-Liceaga, A. F. Fuentes, J. García-
Barriocanal, C. León, and J. Santamaría, J. Solid State Chem. 179, 928 (2006).

${ }^{15}$ A. F. Fuentes, K. Boulahya, M. Maczka, J. Hanuza, and U. Amador, Solid State Sci. 7, 343 (2005).

${ }^{16}$ N. Kim and C. P. Grey, J. Solid State Chem. 175, 110 (2003).

${ }^{17}$ M. P. van Dijk, F. C. Mijlhoff, and A. J. Burggraaf, J. Solid State Chem. 62, 377 (1986).

${ }^{18}$ C. Heremans, B. J. Wuensch, J. K. Stalick, and E. Prince, J. Solid State Chem. 117, 108 (1995).

${ }^{19}$ A. K. Jonscher, Dielectric Relaxation in Solids (Chelsea Dielectric, London, 1983).

${ }^{20}$ K. L. Ngai and R. W. Rendell, ACS Symp. Ser. 679, 45 (1997).

${ }^{21}$ K. L. Ngai and C. León, Phys. Rev. B 60, 9396 (1999).

${ }^{22}$ C. T. Moynihan, Solid State Ionics 105, 175 (1998).

${ }^{23}$ K. Funke, R. D. Banhatti, S. Bruckner, C. Cramer, and D. Wilmer, Solid State Ionics 154-155, 65 (2002); K. Funke and R. D. Banhatti, J. Non-Cryst. Solids 353, 3845 (2007).

${ }^{24}$ K. L. Ngai, Phys. Rev. B 48, 13481 (1993).

${ }^{25}$ R. D. Shannon, Acta Crystallogr., Sect. A: Cryst. Phys., Diffr., Theor. Gen. Crystallogr. 32, 751 (1976).

${ }^{26}$ P. K. Moon and H. L. Tuller, Solid State Ionics, MRS Symposia Proceedings No. 135 (Materials Research Society, Pittsburgh, 1989), p. 149.

${ }^{27}$ H. Yamamura, H. Nishino, K. Kakinuma, and K. Nomura, Solid State Ionics 158, 359 (2003).

${ }^{28}$ T. Van Dijk, K. J. de Vries, and A. J. Burggraaf, Phys. Status Solidi A 58, 115 (1980). 